\title{
Sopra la teoria della inversione di un sistema di funzioni (*).
}

\author{
(del prof. R. Lipschitz, a Bonn.)
}

0 uando in un campo determinato $G$ delle variabili reali $z_{1}, z_{2}, \ldots z_{n}$ è dato un sistema di funzioni $F_{1}, F_{2}, \ldots F_{n}$ reali e ad un sol valore, che hanno le derivate prime parziali, rapporto a queste variabili, finite e continue in tutto il campo, si può dimandare quali condizioni debbono essere sodisfatte affinchè inversamente ad ogni sistema di valori delle funzioni $F_{1}, F_{2}, \ldots F_{n}$ corrisponda un solo sistema di grandezze $z_{1}, z_{2}, \ldots z_{n}$. I concetti, per mezzo dei quali $\mathrm{J}_{\mathrm{A} \text { COB }}$ ha fondato la teoria dei sistemi di funzioni di più variabili sopra la teoria algebrica dei determinanti, conducono alla prima condizione, che il valore del determinante funzionale del sistema di funzioni $F_{1}, F_{2}, \ldots$ $F_{n}$ rapporto al sistema delle variabili $z_{1}, z_{2}, \ldots z_{n}$, in tutto il campo $G$ abbia sempre lo stesso segno.

Per aggiungere a questa altre condizioni, in modo da avere un sistema di condizioni sufficienti perchè la esposta relazione necessariamente debba verificarsi, bisognava mettere in giuoco, con opportune transizioni, il concetto di Riemann della semplice connessione di una varietà.

Ora io svilupperó dapprima un sistema di condizioni che sodisfå alla questione, e poi daro un processo teorico per mezzo del quale possono esser rappresentate le grandezze $z_{1}, z_{2}, \ldots z_{n}$ che corrispondono univocamente a un dato sistema di valori delle funzioni $F_{1}, F_{2}, \ldots F_{n}$. Affinchè questi problemi siano sciolti anche nella supposizione che sia dato un sistema di funzioni $f_{1}, f_{2}, \ldots f_{m}$ delle variabili complesse $y_{1}, y_{2}, \ldots y_{m}$, conviene riguardare, secondo il metodo del sig. Kronecker $\left(^{* *}\right)$, le parti separate delle va-

(*) Dai Nachrichten della Societa delle scienze e dell'Universita di Gottinga (1770, n. 22).

(**) Sopra i sistemi di funzioni di più variabili: Monatsbericht der Berliner Akademie, marzo 1869, pag. 177. 
riabili complesse $y_{1}, y_{2}, \ldots y_{m}$ come variabili reali $z_{1}, z_{2}, \ldots z_{2 m}$ e contemporaneamente le parti separate delle funzioni complesse $f_{1}, f_{2}, \ldots f_{m}$ come funzioni reali $F_{1}, F_{2}, \ldots F_{2 m}$ di quelle $2 m$ variabili reali; allora il metodo prescelto conduce allo scopo.

1.

Il campo $G$ delle variabili reali $z_{1}, z_{2}, \ldots z_{n}$ sia determinato dalla condizione che in esso certe funzioni di queste variabili a un sol valore non possano raggiungere, o rispettivamente non possano superare certi dati valori costanti, e sodisfacciano a questa condizione soltanto valori finiti delle singole variabili $z_{1}, z_{2}, \ldots z_{n}$ : e nel contorno $K$ del campo $G$ quelle funzioni siano rispettivamente uguali a quei dati valori. Se diamo a una funzione $F_{\alpha}$ della serie delle date funzioni $F_{1}, F_{2}, \ldots F_{n}$ un valore ammissibile $G_{\alpha}$, le disuguaglianze $F_{\alpha}-C_{\alpha}>0$, ed $F_{\alpha}-C_{\alpha}<0$ denoteranno due pezzi, nei quali il campo $G$ è diviso dalla equazione $F_{\alpha}-C_{\alpha}=0$. Nel sistema di equazioni :

$$
F_{1}=C_{1}, \quad F_{2}=C_{2}, \ldots \quad F_{n}=C_{n}
$$

sono imposti a tutte le funzioni $F_{\alpha}$ valori costanti $C_{\alpha}$.

Se da questo sistema di equazioni se ne toglie una coppia qualunque $F_{k}=C_{l_{k}}$ ed $F_{l}=C_{l}$, le rimanenti $n-2$ equazioni tra le variabili $z_{1}, z_{2}, \ldots$ $z_{n}$ determinano una varietà del secondo ordine $M_{k, l}$. Il contorno della varietà $M_{l, l}$ sia una varietà di primo ordine chiusa, che appartiene al contorno $K$ del campo $G$.

Ora una rarietà del secondo ordine si dice semplicemente connessa, quando per mezzo di ogni varietà di primo ordine, che si estende semplicemente da un sistema di valori del suo contorno fino ad un altro sistema di valori dello stesso contorno, essa rimane divisa in due pezzi separati (*); ed io suppongo che la varietà $M_{k, l}$ sia semplicemente connessa. Suppongo quindi che nella medesima, tanto la equazione $F_{k}=C_{k}$, quanto la equazione $F_{l}=C_{l}$, ciascuna presa separatamente, rappresenti una varieta di primo or-

(*) Rinmann: Fondamenti di una teoria generale delle funzioni di una variabile complessa: $n^{0} 6$ (Annali di Matematica, serie $1^{\mathrm{a}}$, t. 2). Invece delle espressioni line e superficie sono introdotte nel testo le espressioni: "varieta del primo e del secondo ordine. $n$ 
dine che si estende semplicemente da un sistema di valori della varietà che forma il contorno a un secondo sistema di valori della medesima, e che ambedue queste varietà del primo ordine o abbiano comune un numero finito di sistemi di valori aventi tra loro differenze finite, o coincidano in parti finite, ma che però non possiedano in comune un infinito numero di sistemi di valori discontinui. Ammetto inoltre che la varietà $M_{k, z}$ non possieda singolarità di alcuna specie; in seguito definiremo precisamente questa espressione. Allora, se per ogni sistema di valori nell'intero campo $G$ il determinante funzionale

$$
\mathbf{\Sigma} \pm \frac{\partial F_{1}}{\partial z_{1}} \frac{\partial F_{2}}{\partial z_{2}} \cdots \frac{\partial F_{n}}{\partial z_{n}}=\Delta
$$

e sempre una grandezza dello stesso segno, epperd non è mai zero, si pud dimostrare che al dato sistema di equazioni (1) non puó sodisfare più di un sistema di valori $z_{1}, z_{2}, \ldots z_{n}$.

Una dimostrazione di questo teorema si può dedurre dalla considerazione di una forma analitica, che ho già considerata in un' altra occasione $\left(^{*}\right)$. Alla funzione intera e omogenea di primo grado degli $n$ differenziali $d z_{a}$

$$
a_{1} d z_{1}+a_{2} d z_{2}+\cdots+a_{n} d z_{n}
$$

nella quale $\mathrm{i}$ coefficienti $a_{1}, a_{2}, \ldots a_{n}$ sono funzioni delle variabili $z_{1}, z_{2}, \ldots$ $\boldsymbol{z}_{n}$, reali, a un sol valore e differenziabili, date nel campo $G$, corrisponde la forma bilineare relativamente ad ambedue $\mathrm{i}$ sistemi di differenziali $d z_{\alpha}$ e $\mathrm{d} z_{\beta}$

$$
\mathbf{\Sigma}_{\alpha, \beta}\left(\frac{\partial a_{\alpha}}{\partial z_{\beta}}-\frac{\partial a_{\beta}}{\partial z_{\alpha}}\right)\left(d z_{\alpha} \mathrm{d} z_{\beta}-\mathrm{d} z_{\alpha} d z_{\beta}\right)
$$

la quale per una sostituzione di altre variabili qualunque, invece delle variabili $z_{\alpha}$, rimane covariante alla funzione omogenea (2); per $\alpha$ e $\beta$ si debbono prendere tutte le coppie di numeri differenti della serie da 1 ad $n$. Una determinata applicazione di questa forma (3) serve al presente scopo, e dà contemporaneamente una dimostrazione del teorema che la espressione (2) è un differenziale esatto, quando la forma (3) è identicamente uguale a zero.

${ }^{*}$ ) Ricerche intorno alle funzioni intere $e$ omogenee di $\mathrm{n}$ differenziali: Giornale di Crelle, t. 70, pag. 72. Monatsbericht der Berl. Ak. gennajo 1869, pag. 50.

Annali di Matematica, tomo IV. 
242 Lipschitz: Della inversione di un sistema di funzioni.

Delle $\frac{n(n-1)}{2}$ varietà $M_{k, \imath}$ basta considerarne una sola, e questa sia $M_{n-1, n}$. Se poniamo in generale

$$
d F_{\alpha}=F_{\alpha, 1} d z_{1}+F_{\alpha, 2} d z_{2}+\cdots+F_{\alpha, n} d z_{n}
$$

alla varieta $M_{n-1, n}$ corrispondono le $n-2$ equazioni

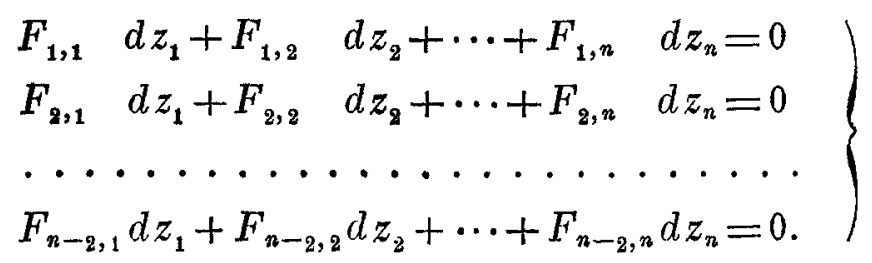

Se $\alpha, \beta, \gamma$ sono numeri qualunque della serie da 1 ad $n$, e si pone

$$
\frac{\partial^{2} \Delta}{\partial F_{n-1, \alpha} \partial F_{n, \beta}^{2}}=(\alpha, \beta)
$$

è noto che si ha $(\alpha, \beta)+(\beta, \alpha)=0$, e dalle (5) si deduce questa equazione fra tre diversi differenziali $d z_{\alpha}, d z_{\beta}, d z_{\gamma}$

$$
(\beta, \gamma) d z_{\alpha}+(\gamma, \alpha) d z_{\beta}+(\alpha, \beta) d z_{\gamma}=0 .
$$

Intorno alle condizioni da verificarsi perchè una delle grandezze $(\alpha, \beta)$ nella varietà $M_{n-1, n}$ possa annullarsi, si parlerà estesamente più sotto. Qui si ammetterà soltanto, che l'annullarsi delle $(\alpha, \beta)$ non possa accadere in alcuna parte di questa varietà, bensi sia possibile in una varietà di primo ordine che giaccia in $M_{n-1, n}$; e che per ogni sistema di valori appartenente alla varietà $M_{n-1, n}$, nel quale $(\alpha, \beta)$ è positivo o negativo, l'unità $\varepsilon_{\alpha, \beta}$ prenda il segno di $(\alpha, \beta)$. La varietà $M_{n-1, n}$ è così fatta che, quando tra le variabili siansi scelte due qualunque $z_{\gamma}, z_{\delta}$, le altre variabili $\boldsymbol{z}_{\alpha}$ divengono tutte dipendenti da queste, dove ad ogni sistema di valori $z_{\gamma}, z_{\delta}$ possono evidentemente corrispondere più sistemi di valori delle variabili $z_{\alpha}$.

Sostituiamo adunque nella forma (3) ad ogni differenziale $d z_{\alpha}, d z_{\beta}$ la espressione $\frac{d z_{\beta}}{d z_{\gamma}} d z_{\gamma}, \frac{d z_{\alpha}}{d z_{\gamma}} d z_{\gamma}$, e ad ogni differenziale $d z_{\alpha}, \mathrm{d} z_{\beta}$ la espressione $\frac{d z_{\alpha}}{d z_{\delta}} d z \delta, \frac{d z_{\beta}}{d z_{\delta}} d z_{\delta}$; moltiplichiamo la forma per la unità $\varepsilon_{\gamma^{\prime} \delta}$ e integriamo sopra una parte $T$ semplicemente connessa della varietà $M_{n-1, n}$, dove gli 
Lipschitz: Della inversione di un sistema di funzioni. 243

aumenti $d z_{\gamma}, d z_{\delta}$ sono presi soltanto positivi; avremo la espressione

$$
I=\iint \mathbf{\Sigma}_{\alpha, \beta}\left(\frac{\partial a_{\alpha}}{\partial z_{\beta}}-\frac{\partial a_{\beta}}{\partial z_{\alpha}}\right)\left(\frac{\partial z_{\alpha}}{\partial z_{\gamma}} \frac{\partial z_{\beta}}{\partial z_{\delta}}-\frac{\partial z_{\alpha}}{\partial z_{\delta}} \frac{\partial z_{\beta}}{\partial z_{\gamma}}\right) \varepsilon_{\gamma, \delta} d z_{\gamma} d z_{\delta}
$$

della discussione della quale dobbiamo occuparci prima d'ogni altra cosa.

I determinanti $\frac{\partial z_{\alpha}}{\partial z_{\gamma}} \frac{\partial z_{\beta}}{\partial z_{\delta}}-\frac{\partial z_{\alpha}}{\partial z_{\delta}} \frac{\partial z_{\beta}}{\partial z_{\gamma}}$ possono esser rappresentati facilmente in modo esplicito, servendosi della equazione (7). Se uno dei numeri $\alpha, \beta$ è uguale ad uno dei numeri $\gamma, \delta$, per es. se $\alpha$ è uguale a $\gamma$, il corrispondente determinante diverrà $\frac{\partial z_{\beta}}{\partial z_{\delta}}$ e la equazione

$$
d z_{\beta}=-\frac{(\delta, \beta)}{(\gamma, \delta)} d z_{\gamma}-\frac{(\beta, \gamma)}{(\gamma, \delta)} d z_{\delta}
$$

darà il risultato

$$
\frac{\partial z_{\beta}}{\partial z_{\delta}}=\frac{(\gamma, \beta)}{(\gamma, \delta)}
$$

Con una ripetuta applicazione del medesimo, si ottiene per ogni coppia di numeri $\alpha, \beta$

$$
\frac{\partial z_{\alpha}}{\partial z_{\gamma}} \frac{\partial z_{\beta}}{\partial z_{\delta}}-\frac{\partial z_{\alpha}}{\partial z_{\delta}} \frac{\partial z_{\beta}}{\partial z_{\gamma}}=\frac{(\alpha, \beta)}{(\gamma, \delta)}
$$

La diretta sostituzione di questi valori trasforma la espressione $I$ nel modo seguente

$$
\left.\begin{array}{l}
I=H, \\
H=\iint \mathbf{\Sigma}_{\alpha, \beta}\left(\frac{\partial a_{\alpha}}{\partial z_{\beta}}-\frac{\partial a_{\beta}}{\partial z_{\alpha}}\right)(\alpha, \beta) \frac{\varepsilon_{\gamma, \delta}}{(\gamma, \delta)} d z_{\gamma} d z_{\delta} .
\end{array}\right\}
$$

Se invece si scioglie $I$ in una somma d'integrali, e nell'integrale

$$
\iint\left(\frac{\partial a_{\alpha}}{\partial z_{\beta}}-\frac{\partial a_{\beta}}{\partial z_{\alpha}}\right)\left(\frac{\partial z_{\alpha}}{\partial z_{\gamma}} \frac{\partial z_{\beta}}{\partial z_{\delta}}-\frac{\partial z_{\alpha}}{\partial z_{\delta}} \frac{\partial z_{\beta}}{\partial z_{\gamma}}\right) \varepsilon_{\gamma, \delta} d z_{\gamma} d z_{\delta}
$$

s'introducono le variabili $z_{\alpha}, z_{\beta}$ come variabili indipendenti della integrazione, siccome per la $\left(9^{*}\right)$ il determinante della sostituzione ha il segno $\varepsilon_{\alpha, \beta} \varepsilon_{\gamma, \delta}$, cosi questo integrale diviene

$$
\iint\left(\frac{\partial a_{\alpha}}{\partial z_{\beta}}-\frac{\partial a_{\beta}}{\partial z_{\alpha}}\right) \varepsilon_{\alpha, \beta} d z_{\alpha} d z_{\beta}
$$


e si ha la equazione

$$
\left.\begin{array}{l}
I=L, \\
L=\mathbf{\Sigma}_{\alpha, \beta} \iint\left(\frac{\partial a_{\alpha}}{\partial z_{\beta}}-\frac{\partial a_{\beta}}{\partial z_{\alpha}}\right) \varepsilon_{\alpha, \beta} d z_{\alpha} d z_{\beta} .
\end{array}\right\}
$$

Questa dimostra che il valore della espressione $I$ è indipendente affatto dalla scelta delle variabili $z_{\gamma}, z_{\delta}$.

Poichè dalla equazione $(\alpha, \beta)+(\beta, \alpha)=0$ segue l'altra $\varepsilon_{\alpha, \beta}+\varepsilon_{\beta, \alpha}=0$, si ha

$$
\left(\frac{\partial \alpha_{\alpha}}{\partial z_{\beta}}-\frac{\partial \alpha_{\beta}}{\mathrm{c} z_{\alpha}}\right) \varepsilon_{\alpha, \beta}=\frac{\partial \alpha_{\alpha}}{\partial z_{\beta}} \varepsilon_{\alpha, \beta}+\frac{\partial \alpha_{\beta}}{\partial z_{\alpha}} \varepsilon_{\beta, \alpha}
$$

e quindi :

$$
\left.\begin{array}{r}
L=\mathbf{\Sigma}_{\alpha} \iint\left(\frac{\partial \alpha_{\alpha}}{\partial z_{\alpha+1}} \varepsilon_{\alpha, \alpha+1} d z_{\alpha} d z_{\alpha+1}+\frac{\partial a_{\alpha}}{\partial z_{\alpha+2}} \varepsilon_{\alpha, \alpha+2} d z_{\alpha} d z_{\alpha+2}+\cdots+\right. \\
\left.\frac{\partial \alpha_{\alpha}}{\partial z_{\alpha+n-1}} \varepsilon_{\alpha, \alpha+n-1} d z_{\alpha} d z_{\alpha+n-1}\right)
\end{array}\right\}
$$

dove si deve sottrarre il numero $n$ da tutti gl'indici maggiori di $n$. La introduzione delle yariabili indipendenti d'integrazione $z_{\alpha}, z_{\alpha_{+1}}$ dà la equazionè :

$$
\left.\begin{array}{l}
\iint\left(\frac{\partial a_{\alpha}}{\partial z_{\alpha+1}} \varepsilon_{\alpha, \alpha+1} d z_{\alpha} d z_{\alpha+1}+\cdots+\frac{\partial a_{\alpha}}{\partial z_{\alpha+n-1}} \varepsilon_{\alpha, \alpha+n-1} d z_{\alpha} d z_{\alpha+n-1}\right) \\
=\iint\left(\frac{\partial a_{\alpha}}{\partial z_{\alpha+1}}+\frac{\partial a_{\alpha}}{\partial z_{\alpha+2}} \frac{\partial z_{\alpha+2}}{\partial z_{\alpha+1}}+\cdots+\frac{\partial a_{\alpha}}{\partial z_{\alpha+n-1}} \frac{\partial z_{\alpha+n-1}}{\partial z_{\alpha+1}}\right) \varepsilon_{\alpha, \alpha+1} d z_{\alpha} d z_{\alpha+1} \cdot
\end{array}\right)
$$

e quindi

$$
L=\Sigma_{\alpha} \iint\left(\frac{\partial a_{\alpha}}{\partial z_{\alpha+1}}+\frac{\partial \alpha_{\alpha}}{\partial z_{\alpha+2}} \frac{\partial z_{\alpha_{+2}}}{\partial z_{\alpha_{+1}}}+\cdots+\frac{\partial a_{\alpha}}{\partial z_{\alpha+n-1}} \frac{\partial z_{\alpha+n-1}}{\partial z_{\alpha+1}}\right) \varepsilon_{\alpha, \alpha+1} d z_{\alpha} d z_{\alpha_{+1}}
$$

La espressione tra parentesi è la derivata completa della funzione $a_{a}$ rapporto alla variabile $z_{\alpha_{+1}}$, per un valore costante di $z_{\alpha}$. Dunque, se nella integrazione da effettuarsi relativamente all' elemento $d z_{\alpha} d z_{\alpha+1}$ si dà un valore costante alla variabile $z_{\alpha}$, si puó eseguire immediatamente la integrazione indefinita rispetto alla variabile $z_{\alpha_{+1}}$ in quell' intervallo della stessa, in cui la grandezza $\varepsilon_{\alpha, \alpha_{+1}}$ rimane invariata. I valori delle funzioni ottenute colla integrazione indefinita si devono prendere negativi nei posti dove la variabile $z_{\alpha_{+1}}$ entra nel campo della integrazione, e positivi nei posti dove 
Lipschitz: Della inversione di un sistema di funzioni. 245

ne esce. Siccome le variabili in questa integrazione hanno soltanto incrementi positivi, cosi, purchè la variabile $z_{\alpha_{+1}}$ si muova sempre verso l'interno del campo della integrazione, per ogni posto d'ingresso $d z_{\alpha_{+1}}$ è una quantità positiva, e per ogni posto di egresso una quantità negativa. Ora bisogua esaminare accuratamente i limiti degl' intervalli per la variabile $z_{\alpha+1}$, i quali si trovano in parte sul contorno della varieta $T$, e in parte nelle varietà di primo ordine nelle quali la grandezza $(\alpha, \alpha+1)$ è uguale a zero.

Il contorno della varietà $T$ sia determinato in modo che nell'interno di $T$ certe funzioni a un sol valore delle variabili $z_{1}, z_{2}, \ldots z_{n}$ possano ottenere soltanto valori positivi; quando si considerano successivamente le diverse parti del contorno, denoteremo sempre $\operatorname{con} \Theta$ la funzione relativa. Allora, nel passaggio da un sistema di valori del contorno nell'interno della varietà $T$, il differenziale $d \Theta$ è una grandezza positiva. Aggiungendo al sistema delle equazioni (5) la equazione

$$
d \Theta=\Theta_{1} d z_{1}+\Theta_{2} d z_{2}+\cdots+\Theta_{n} d z_{n}
$$

ed eliminando $n-2$ differenziali, si ha

$$
R_{\alpha_{+1}} d z_{\alpha}-R_{\alpha} d z_{\alpha_{+1}}=(\alpha, \alpha+1) d \Theta
$$

Ora, siccome nella integrazione che si deve effettuare, il valore indefinito della variabile $z_{\alpha}$ rimane costante, e quindi $d z_{\alpha}=0$, per il differenziale $d z_{\alpha_{+1}}$ abbiamo

$$
R_{\alpha} d z_{\alpha_{+1}}=-(\alpha, \alpha+1) d \Theta
$$

dove $R_{\alpha}$ non puó essere uguale a zero. Se denotiamo il segno di questa grandezza con $\varepsilon_{\alpha}$, il differenziale $d z_{\alpha_{+1}}$ per un moto della variabile $z_{\alpha_{+1}}$ diretto verso l'interno del campo della integrazione ha il segno $-\varepsilon_{\alpha} \varepsilon_{\alpha, \alpha_{+1}}$. Dunque per cid che abbiamo detto, ogni posto d'ingresso è caratterizzato dalla equazione $\varepsilon_{\alpha} \varepsilon_{\alpha, \alpha_{+1}}=-1$, e ogni posto di egresso dalla equazione $\varepsilon_{\alpha} \varepsilon_{\alpha, \alpha_{+1}}=+1$, e la funzione $\varepsilon_{\alpha, \alpha+1} a_{\alpha}$ ottenuta dalla (14) colla integrazione indefinita rispetto alla variabile $z_{\alpha+1}$ acquista in ogni posto del contorno della varietà $T$ il valore $\varepsilon_{\alpha} a_{\alpha}$.

Resta ora da stabilirsi come pud annullarsi la grandezza $(\alpha, \alpha+1)$ nella varietà $M_{n-1, n}$ in una varietà del primo ordine. Fra il differenziale di una variabile dipendente $z_{\gamma}$ e i differenziali delle due variabili indipendenti $z_{\alpha}$ e $z_{\alpha+1}$, si ha dalla (7) la equazione

$$
(\alpha+1, \gamma) d z_{\alpha}+(\gamma, \alpha) d z_{\alpha+1}+(\alpha, \alpha+1) d z_{\gamma}=0 .
$$


Siccome nella integrazione rapporto a $z_{x+1}$, è il differenziale $d z_{u}=0$, si ha la equazione

$$
(\gamma, \alpha) d z_{\alpha_{+1}}+(\alpha, \alpha+1) d z_{\gamma}=0
$$

dalla quale $d z_{\gamma}$ è pienamente determinato finchè la grandezza $(\alpha, \alpha+1)$ è differente da zero. Perciò, finchè questa condizione è sodisfatta, da ogni sistema di valori $\left(z_{1}, z_{2}, \ldots z_{n}\right)$, se $z_{\alpha}$ rimane costante e $z_{\alpha+1}$ si aumenta di $d z_{\alpha_{+1}}$, si perviene ad un secondo sistema di valori pienamente determinato. Sopra abbiamo osservato, che quando la varietà $M_{n-1, n}$ è riferita alle variabili indipendenti $z_{\alpha}, z_{\alpha+1}$, ad ogni sistema di valori $z_{\alpha}, z_{\alpha+1}$ possono appartenere più sistemi di valori dell'altra variabile $z_{\gamma}$, e da cio che ora abbiamo detto consegue che il coincidere di più di tali sistemi di valori puó solo accadere là dove la grandezza $(\alpha, \alpha+1)$ si annulla. L'annullarsi in una parte della varietà $M_{n-1, n}$ è già stato escluso. Per le varietà di primo ordine, nelle quali $(\alpha, \alpha+1)$ si annulla, io ammetto che, se ad una tal varietà appartiene la combinazione di valori $z_{\alpha}, z_{\alpha_{+1}}$, per un movimento diretto verso l'interno della varietà $M_{n-1, n}$ col valore di $z_{\alpha}$ tenuto costante, la variabile $z_{\alpha_{+1}}$ possa ottenere soltanto un aumento $d z_{\alpha_{+1}}$ di dato segno; che a questo movimento della variabile $z_{\alpha_{+1}}$ corrisponda il passaggio da un determinato sistema di valori $z_{\gamma}$ a due sistemi di valori $z_{\gamma}$ differenti tra loro; che la grandezza $(\alpha, \alpha+1)$ sia positiva per uno di questi sistemi di valori, negativa per l'altro; e che la integrazione della espressione $\frac{d z_{\alpha+1}}{(\alpha, \alpha+1)}$ estesa da questo sistema di valori a un altro che abbia una differenza finita con esso, offra un significato preciso. Queste condizioni definiscono la espressione usata sopra, che la varietà $M_{n-1, n}$ non possa contenere nessuna specie di singolarità. Un semplice esempio pel prescritto comportarsi di una varietà del secondo ordine ci è dato, per $n=3$, da $F_{1}=\frac{z_{1}^{2}}{b_{1}}+\frac{z_{2}^{2}}{b_{2}}+\frac{z_{3}^{2}}{b_{3}}$, dove $b_{1}, b_{2}, b_{3}$ sono costanti positive; la equazione $F_{1}=C_{1}$ rappresenta allora la varietà $M_{2,3}$. Le esposte condizioni generali rimuovono il dubbio che può nascere, nella eseguita trasformazione di integrali doppi, dallo annullarsi delle grandezze $(\gamma, \partial)$ nel denominatore. Queste condizioni producono inoltre nella integrazione da eseguirsi nell' interno della varietà $T$ rapporto alla variabile $z_{\alpha_{+1}}$, mantenendo costante $z_{\alpha}$, il seguente effetto. Ciascuna varietà di primo ordine contenuta in $T$, nella quale la grandezza $(\alpha, \alpha+1)$ si annulli, è jl limite di due campi in uno dei quali $\varepsilon_{\alpha, \alpha_{+1}}=1$, e nell'altro 
$\varepsilon_{\alpha, \alpha_{+1}}=-1$. Ogni sistema di valori di questa varietà dí primo ordine, per $z_{\alpha}$ costante, forma per la variabile $z_{\alpha+1}$ in ambedue i campi contemporaneamente 0 un punto d'ingresso 0 un punto di egresso, perchè l'aumento $d \boldsymbol{z}_{\alpha_{+1}}$ ha in ambedue i campi lo stesso segnno. Quindi, per la integrazione relativa alla variabile $z_{\alpha_{+1}}$, nei sistemi di valori della suddetta varietà si presentano sempre ambedue $\mathrm{i}$ valori della funzione $+a_{\alpha}$ e $-a_{\alpha}$ che reciprocamente si distruggono. Percio il risultato finale contiene soltanto i valori della funzione corrispondenti al contorno della varietà $T$, precedentemente determinati, e prende questa forma (*)

$$
\left.\begin{array}{l}
I=N, \\
N=\int\left(a_{1} \varepsilon_{1} d z_{1}+a_{2} \varepsilon_{2} d z_{y}+\cdots+a_{n} \varepsilon_{n} d z_{n}\right) .
\end{array}\right\}
$$

Qui bisogna fare una osservazione rispetto agli $n$ segni $\varepsilon_{\alpha}$. Se per $\Phi$ si intende una funzione a un sol valore delle variabili $z_{1}, z_{2}, \ldots z_{n}$ e si formano le $n$ equazioni

$$
\left.\begin{array}{llll}
F_{1,1} & d z_{1}+F_{12} & d z_{2}+\cdots+F_{1, n} & d z_{n}=0 \\
F_{2,1} & d z_{1}+F_{22} & d z_{2}+\cdots+F_{2, n} & d z_{n}=0 \\
\cdots \ldots \ldots \ldots \ldots \ldots & \ldots \ldots \ldots+\ldots \\
F_{n-2,1} & d z_{1}+F_{n-2, n} d z_{2}+\cdots+F_{n-2, n} d z_{n}=0 \\
\Theta_{1} & d z_{1}+\Theta_{2} & d z_{2}+\cdots+\Theta_{n} & d z_{n}=0 \\
\Phi_{1} & d z_{1}+\Phi_{2} & d z_{2}+\cdots+\Phi_{n} & d z_{n}=d \Phi
\end{array}\right\}
$$

e se $R$ è il determinante delle $n^{2}$ quantità dei primi membri, ed è differente da zero, risolvendo le equazioni abbiamo

$$
R d z_{\alpha}=R_{\alpha} d \Phi \text {. }
$$

Da queste sono determinati gli $n$ differenziali $d z_{\alpha}$ per l'unico differenziale $d \Phi$, e i segni degli $n$ differenziali $d z_{\alpha}$ si ottengono da quelli $\varepsilon_{a}$ delle $R_{a}$ e da quelli delle grandezze $R$ e $d \Phi$. Se fissiamo che il differenziale $d \Phi$ abbia il segno del determinante $R$, ogni differenziale $d z_{\alpha}$ avrà sul contorno della

(*) Il risultato per il caso $n=2$ è dato da RiEmANn nella citata memoria: Fondamenti ecc., pag. 9, e nell'altra: Lehrsätze aus der Analysis situs ete. (Giornale di Crelle, t. 54, pag. 105). Il risultato per il caso $n=3$ si trova nel lavoro di H. Hankel: Sulla teoria generale del moto dei fuidi, Gottinga 1861 ; di questo lavoro non ho però cognizione che per un estratto inserito nei Fortschritte der Physik, 1861, pag. 57. 
varietà $T$, a cui le equazioni (20) si riferiscono, il segno di $R_{\alpha}$. Questa determinazioce dà il senso in cui un sistema di valori percorre la relativa varietà chiusa di primo ordine, e coincide colla determinazione fornita dal principio di passaggio, sviluppato dal sig. Kronecker (*). L'elemento dell' integrale semplice $N$ è la forma lineare (2), l'elemento dell'integrale doppio è la forma bilineare (3) covariante alla forma (2), e la relazione (19) esprime l' eguaglianza dei due integrali.

2.

Ora faremo una doppia applicazione della relazione (19). Per la prima applicazione supporremo che la forma (3) si annulli identicamenre, ossia che in tutto il campo $G$ siano sodisfatte per la forma (2) le $\frac{n(n-1)}{2}$ condizioni d'integrabilità

$$
\frac{\partial a_{\alpha}}{\partial z_{\beta}}-\frac{\partial a_{\beta}}{\partial z_{\alpha}}=0
$$

Siccome in conseguenza di cio la espressione $I$ è uguale a zero, dalla (19) arremo la equazione

$$
N=\int\left(a_{1} \varepsilon_{1} d z_{1}+a_{2} \varepsilon_{2} d z_{2}+\cdots+a_{n} \varepsilon_{n} d z_{n}\right)=0 .
$$

Nel contorno della varieta $T$ semplicemente connessa prendiamo un sistema fisso di valori $\left[z_{1}(0), z_{2}(0), \ldots z_{n}(0)\right]$ e un sistema di valori mobile $\left(z_{1}, z_{2}, \ldots z_{n}\right)$ e chiamiamo $S$ ed $S^{\prime}$ le due parti nelle quali è spezzato il contorno da questi due sistemi di valori. La parte $S$ sia quella che cresce quando alle variabili $z_{1}, z_{2}, \ldots z_{n}$ del sistema mobile si danno gli aumenti $\varepsilon_{1} d z_{1}, \varepsilon_{2} d z_{2}, \ldots \varepsilon_{n} d z_{n}$. Allora l'equazione (23) mostra che l'integrale

$$
\int\left(\varepsilon_{1} a_{1} d z_{1}+\varepsilon_{2} a_{2} d z_{2}+\cdots+\varepsilon_{n} a_{n} d z_{n}\right)
$$

esteso alla parte $S$ e l'integrale

$$
-\int\left(\varepsilon_{1} a_{1} d z_{1}+\varepsilon_{2} a_{2} d z_{2}+\cdots+\varepsilon_{n} a_{n} d z_{n}\right)
$$

(*) Nella memoria citata, pag. 160 . 
esteso alla parte $S^{\prime}$ sono tra loro uguali. Se un sistema di valori $\left(\zeta_{1}, \zeta_{2}, \ldots\right.$ $\left.\zeta_{n}\right)$ percorre la parte $S$ dal sistema $\left[z_{1}(0), z_{2}(0), \ldots z_{n}(0)\right]$ sino al sistema $\left(z_{1}, z_{2}, \ldots z_{n}\right)$, ogni variabile $\zeta_{\alpha}$ riceve l'aumento $\varepsilon_{\alpha} d \zeta_{\alpha}$; se un sistema di valori $\left(\zeta_{1}, \zeta_{2}, \ldots \zeta_{n}\right)$ percorre la parte $S^{\prime}$ dal sistema $\left[z_{1}(0), z_{2}(0), \ldots z_{n}(0)\right]$ sino al sistema $\left(z_{1}, z_{2}, \ldots z_{n}\right)$, ogni variabile $\zeta_{\alpha}$ riceve l'aumento $-\varepsilon_{\alpha} d \zeta_{\alpha}$. Il valore dell'integrale (23*) è quindi indipendente dalla varietả del primo ordine sopra cui si estende l'integrale stesso dal sistema di valori $\left[z_{1}(0)\right.$, $\left.z_{2}(0), \ldots z_{n}(0)\right]$ sino al sistema $\left(z_{1}, z_{2}, \ldots z_{n}\right)$, e È quindi una funzione delle variabili $z_{1}, z_{2}, \ldots z_{n}$, che si annulla quando per ogni valore di $\alpha$ sia $z_{\alpha}=z_{\alpha}(0)$. Se con $*$ s'intende una grandezza data, positiva, piccola quanto si vuole, e con $\eta_{\alpha}$ una frazione propria, positiva o negativa, si potrà a cagione della continuità della funzione $a_{a}$ per ogni sistema di valori $\left(z_{1}\right.$, $z_{2}, \ldots z_{n}$ ) dare a una variabile $z_{\alpha}$ un tale aumento $\zeta_{\alpha}$, , non mutando le altre variabili, che si abbia

$$
\mathbb{E}\left(z_{\alpha}+\zeta_{\alpha}\right)-E\left(z_{\alpha}\right)=\left(a_{\alpha}+\eta_{\alpha} z\right) \ddot{\zeta}_{\alpha} .
$$

Questa, passando al limite, ci dice che la derivata della funzione $\leftleftarrows$ rapporto alla variabile $\boldsymbol{z}_{\alpha}$ ̀े uguale alla grandezza $a_{\alpha}$, e mostra cosi, come doveva essere, la integrabilità della espressione (2) nella supposizione delle equazioni (22).

Se è data una funzione $\mathfrak{F}$ delle variabili $z_{1}, z_{2}, \ldots z_{n}$, la quale per il sistema di valori $z_{\alpha}=z_{\alpha}(0)$ diviene uguale a zero, e le cui derivate prese rispetto a $z_{\alpha}$ sono uguali rispettivamente alle quantita $a_{\alpha}$, e se può darsi per ogni sistema di valori $\left(z_{1}, z_{2}, \ldots z_{n}\right)$ del campo $G$ a ciascuna variabile $z_{\alpha}$ un aumento $\zeta_{\alpha}$ indipendente, quanto alla sua grandezza, da questo sistema di valori, il quale, essendo $x$ una grandezza positiva, data, piccola quanto si vuole, corrisponde alla equazione

$$
\mathfrak{F}\left(z_{\alpha}+\zeta_{\alpha}\right)-\mathfrak{F}\left(z_{\alpha}\right)=\left(\alpha_{\alpha}+\eta_{\alpha} x\right) \zeta_{\alpha}
$$

si può dimostrare che questa funzione $\mathfrak{F}$ è identica colla funzione sopra definita . Il mio amico e collega Kortum verbalmente mi ha fatto avvertire, che la condizione (24*), necessaria per la dimostrazione, dev'essere espressamente formulata, poichè la equazione $\frac{\partial \mathscr{F}}{\partial z_{\alpha}}=a_{\alpha}$ puo sussistere benissimo, senza che questa condizione sia sodisfatta. Questa riflessione è di natura molto affine a quelle fatte dal sig. Heine nella memoria Sopra le serie trigonometriche, Giornale di Crelle-Borchardt, t. 71, pag. 353. 
Per tutte le funzioni $F_{\gamma}$ del dato sistema supporremo percio che la equazione

$$
F_{\gamma}\left(z_{\alpha}+\zeta_{\alpha}\right)-F_{\gamma}\left(z_{\alpha}\right)=\left(\frac{\partial F_{\gamma}}{\partial z_{\alpha}}+\eta_{\alpha} x\right) \zeta_{\alpha}
$$

possa essere sodisfatta nel modo dichiarato. Allora la espressione (23*), ammesso che sia

$$
a_{\alpha}=\frac{\partial F_{n-1}}{\partial z_{\alpha}}
$$

dà l'equazione

$$
F_{n-1}\left(z_{1}, z_{2}, \ldots z_{n}\right)-F_{n-1}\left(z_{1}(0), z_{2}(0), \ldots z_{n}(0)=\sum_{\alpha} \int \frac{\partial F_{n-1}}{\partial z_{\alpha}} \varepsilon_{\alpha} d z_{\alpha} .\right.
$$

La corrispondente supposizione vale anche per la effettuazione di ogni integrazione definita, e quindi anche per quella che si fa per ottenere la equazione (19).

La seconda applicazione della equazione (19) si ottiene prendendo per le funzioni $a_{\alpha}$

$$
a_{\alpha}=\frac{\partial F_{n-1}}{\partial z_{\alpha}} F_{n}
$$

In conseguenza di questa determinazione, si ha

$$
\frac{\partial \alpha_{\alpha}}{\partial z_{\beta}}-\frac{\partial \alpha_{\beta}}{\partial z_{\alpha}}=\frac{\partial F_{n-1}}{\partial z_{\alpha}} \frac{\partial F_{n}}{\partial z_{\beta}}-\frac{\partial F_{n-1}}{\partial z_{\beta}} \frac{\partial F_{n}}{\partial z_{\alpha}}
$$

e la somma che bisogna prendere nella (10) rispetto agl'indici $\alpha$ e $\beta$ diviene uguale al determinante funzionale del sistema $F_{1}, F_{2}, \ldots F_{n}$

$$
\Sigma_{\alpha, \beta}\left(\frac{\partial a_{\alpha}}{\partial z_{\beta}}-\frac{\partial a_{\beta}}{\partial z_{\alpha}}\right)(\alpha, \beta)=\Delta
$$

Sostituendo questa espressione nell'integrale $H$, e la espressione (26) nell'integrale $N$, le (10) e (19) danno la equazione

$$
\iint \Delta \frac{\varepsilon_{\gamma, \delta}}{(\gamma, \delta)} d z_{\gamma} d z_{\delta}=\int F_{n} \Sigma_{\alpha} \frac{\partial F_{n-1}}{\partial z_{\alpha}} \varepsilon_{\alpha} d z_{\alpha}
$$

La deduzione della equazione (19) richiede, come abbiamo precedentemente osservato, la supposizione che allora abbiamo fatto. Poichè ora si applica la sostituzione (26), dovranno sodisfare a questa supposizione tanto le derivate prime quanto le seconde delle funzioni $F_{\gamma}$. 
Lipschitz: Della inversione di un sistema di funzioni. 251

La equazione (28) nel caso $n=2$ si riduce alla

$$
\iint\left(\frac{\partial F_{1}}{\partial z_{1}} \frac{\partial F_{2}}{\partial z_{2}}-\frac{\partial F_{1}}{\partial z_{2}} \frac{\partial F_{2}}{\partial z_{2}}\right) d z_{1} d z_{2}=\int F_{2}\left(\frac{\partial F_{1}}{\partial z_{1}} \varepsilon_{1} d z_{1}+\frac{\partial F_{2}}{\partial z_{2}} \varepsilon_{2} d z_{2}\right)
$$

stabilita da Riemann nella memoria: Sopra le superficie di area minima entro un dato contorno, pag. 12. Un assiduo studio di questa equazione mi ha condotto alla dimostrazione del teorema sopra enunciato, che adesso passo ad esporre.

3.

Supponiamo che esistano due sistemi di valori differenti tra loro $\left[z_{1}(0)\right.$, $\left.z_{2}(0), \ldots z_{n}(0)\right]$, ossia $W(0)$, e $\left[z_{1}(1), z_{2}(1), \ldots z_{n}(1)\right]$, ossia $W(1)$, che sodisfacciano alle $n$ equazioni (1)

$$
F_{1}=C_{1}, F_{2}=C_{2}, \ldots F_{n}=C_{n} \text {. }
$$

Consideriamo ora una qualunque della varietà $M_{k, l}$ e questa sia sempre la varietå $M_{n-1, n}$. Secondo la fatta supposizione, sia essa semplicemente connessa, e nella medesima tanto la equazione $F_{n-1}=C_{n-1}$ quanto la equazione $F_{n}=G_{n}$ rappresentino varietả di primo ordine, che da un sistema di valori della varietâ formante il contorno si estendano semplicemente sino a un altro sistema di valori dello stesso contorno. Quindi $M_{n-1, n}$ rimarrà divisa, tanto dalla varietà di primo ordine $F_{n-1}=G_{n-1}$, quanto dalla varietà di primo ordine $F_{n}=C_{n}$, in due varietà semplicemente connesse (*), le quali sono caratterizzate rispettivamente dalle disuguaglianze $F_{n-1}-C_{n-1}>0$, $F_{n-1}-G_{n-1}<0$ ed $F_{n}-C_{n}>0, F_{n}-C_{n}<0$. I sistemi di valori $W(0)$ e $W(1)$ appartengono ciascuno ad ambedue le varieta $F_{n-1}=C_{n-1}$ ed $F_{n}=C_{n}$, ed in ambedue il passaggio dal sistema di valori $W(0)$ al sistema di valori $W(1)$ è possibile in un sol modo. Per la supposizione fatta, le varietà $F_{n-1}=C_{n-1}$ ed $F_{n}=C_{n}$ hanno in comune nella varietà $M_{n-1, n} 0$ un numero finito di sistemi di valori differenti tra loro, o esse coincidono completamente in parti finite. Bisogna considerare separatamente questi due casi possibili.

Nel primo caso, sia $W$ il primo sistema di valori nel quale le varietå di primo ordine $F_{n-1}=C_{n-1}$ ed $F_{n}=C_{n}$ s'incontrano muovendosi, nel modo che

(*) Riemanin: Fondamenti, ecc. n. 6 . 
abbiamo detto, a partire da $W(0)$. Denotiamo con $S$ la parte della varietá $F_{n-1}=G_{n-1}$ che si estende da $W(0)$ a $W$, e con $S^{\prime}$ la parte della varietà $F_{n}=C_{n}$ che si estende da $W(0)$ a $W$. Allora $S$ ed $S^{\prime}$ formeranno il contorno di una parte finita, semplicemente connessa, della varietà $M_{n-1}$, e riferiamo a questa parte $T$ la equazione (28). In questa equazione l'integrale $\int F_{n} \Sigma_{\alpha} \frac{\partial F_{n-1}}{\partial z_{\alpha}} \varepsilon_{\alpha} d z_{\alpha}$ è composto di due parti, la prima delle quali è estesa ad $S$, la seconda ad $S^{\prime}$. Il primo integrale esteso ad $S$ ha il valore zero, perchè, secondo la definizione, per ogni sistema di valori di $S$ l'aggregato $\Sigma_{\alpha} \frac{\partial F_{n-1}}{\partial z_{\alpha}} \varepsilon_{\alpha} d z_{\alpha}$ si annulla. Nel secondo integrale esteso ad $S^{\prime}$ la funzione $F_{n}$ ha il valore costante $G_{n}$, quindi

$$
\int F_{n} \mathbf{\Sigma}_{\alpha} \frac{\partial F_{n-1}}{\partial z_{\alpha}} \varepsilon_{\alpha} d z_{\alpha}=C_{n} \int \mathbf{\Sigma}_{\alpha} \frac{\partial F_{n-1}}{\partial z_{\alpha}} \varepsilon_{\alpha} d z_{\alpha}
$$

L'integrale $\int \mathbf{\Sigma}_{\alpha} \frac{\partial F_{n-1}}{\partial z_{\alpha}} \varepsilon_{\alpha} d z_{\alpha}$ è, a cagione della $\left(25^{*}\right)$, uguale alla differenza dei valori della funzione $F_{n-1}$ per i valori $W$ e $W(0)$ epperd, nelle vigenti relazioni, uguale a zero. Dunque si annulla anche il secondo integrale e quindi anche tutto il valore del primo membro della (28). Per conseguenza, colle supposizioni fatte, si ha la equazione

$$
\iint \Delta \frac{\varepsilon_{\gamma, \delta} d z_{\gamma} d z_{\delta}}{(\gamma, \delta)}=0
$$

Fin qui non ei siamo serviti di alcuna supposizione speciale rispetto al determinante funzionale $\Delta$. Ma si deve ammettere che $\Delta$ in tutto il campo $G$, e quindi anche in tutta la varietà $T$, non muti mai segno. Ora poichè nel precedente integrale l' elemento $\frac{\varepsilon_{\gamma, \delta}}{(\gamma, \delta)} d z_{\gamma} d z_{\delta}$ è sempre positivo, e la grandezza $\Delta$ sempre positiva o sempre negativa, il valore dell'integrale non può annullarsi, e la equazione (30) contiene una contraddizione. Dunque l'ipotesi che le equazioni (1) siano sodisfatte da ambedue i sistemi di valori $W(0)$ e $W(1)$, e incompatibile colla supposizione che in $M_{n-1, n}$ lo varietà di primo ordine $F_{n-1}=C_{n-1}$ ed $F_{n}=C_{n}$ abbiano in comune un numero finito di sistemi di valori finiti, differenti tra loro.

Il secondo caso che deve considerarsi quanto ai sistemi di valori $W(0)$, $W(1)$, è quello in cui le varietà di primo ordine $F_{n-1}=C_{n-1}$ ed $F_{n}=C_{n}$ 
Lipschitz: Della inversione di un sistema di funzioni. 253

contenute in $M_{n-1, n}$, in un moto eseguito da $W(0)$ a $W(1)$, coincidano in una parte finita. $\hat{\mathrm{E}}$ chiaro che per questa parte i differenziali totali delle funzioni $F_{\alpha}$ dovrebbero allora essere tutti uguali a zero. Ma dalle equazioni

$$
\begin{aligned}
& F_{1,1} d z_{1}+F_{1,2} d z_{2}+\cdots+F_{1, n} d z_{n}=0 \\
& F_{2,1} d z_{1}+F_{2,2} d z_{2}+\cdots+F_{2, n} d z_{n}=0 \\
& F_{n, 1} d z_{1}+F_{n, 2} d z_{2}+\cdots+F_{n, n} d z_{n}=0
\end{aligned}
$$

segue l'annullarsi del determinante funzionale $\Delta$, che per supposizione è escluso. Dunque questo secondo caso non può aver luogo, come non puó aver luogo il primo, e rimane percid dimostrata la proposizione, che le $n$ equazioni (1), sotto le condizioni stabilite, possono essere sodisfatte soltanto da un unico sistema di valori $z_{1}, z_{2}, \ldots z_{n}$.

Se riguardiamo con Rremann le $n$ variabili $z_{1}, z_{2}, \ldots z_{n}$ come le coordinate di un punto in una varietà $n$-upla [Sopra le ipotesi che servono di fondamento alla geometria (*)], ne risulta che la presente ricerca non contiene il concetto dell'elemento lineare di questa varietá. Le sopra accennate deduzioni di Riemann della equazione (19) nel caso $n=2$ mostrano, confrontate sotto questo punto di vista, una notevole differenza. L'integrale denotato con $N$ nella (19) è riferito nel primo lavoro all'elemento lineare della curva che forma il contorno del pezzo di superficie $T$, al contrario nel secondo lavoro è riguardato come l'integrale di una espressione di due differenziali.

4.

Quando si verificano le condizioni, sotto le quali le equazioni

$$
\left.\begin{array}{l}
F_{1}\left(z_{1}, z_{2}, \ldots z_{n}\right)=X_{1} \\
F_{2}\left(z_{1}, z_{2}, \ldots z_{n}\right)=X_{2} \\
\ldots \ldots \ldots \ldots . . . . \\
F_{n}\left(z_{1}, z_{2}, \ldots z_{n}\right)=X_{n}
\end{array}\right\}
$$

(*) Annali di Matematica, serie $2^{\mathrm{a}}$, t. $3^{\circ}$, pag. 315 . 
possono essere sodisfatte da un solo sistema di valori $\left(z_{1}, z_{2}, \ldots z_{n}\right)$, la determinazione di questo sistema di valori può effettuarsi nel modo seguente. Sostituendo il sistema speciale di valori $z_{\alpha}=z_{\alpha}(0)$, si ha il sistema di equazioni

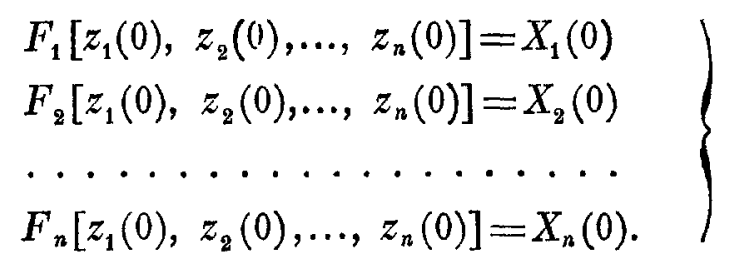

Si dimanda prima qual'è il sistema di valori $\left[z_{1}(1), z_{2}(1), \ldots z_{n}(1)\right]$ il quale sodisfà alle $n$ equazioni

$$
F_{1}=X_{1}(0), F_{2}=X_{2}(0), \ldots F_{n-1}=X_{n-1}(0), F_{n}=X_{n} .
$$

La totalitá dei sistemi di valori per i quali tutte le funzioni $F_{\beta}$, ad eccezione di $F_{n}$, prendono rispettivamente i valori fissi $X_{\beta}(0)$, mentre al contrario $F_{n}$ percorre con continuita i valori da $X_{n}(0)$ a $X_{n}$, forma una semplice varietà di primo ordine, ed è caratterizzata dall'annullarsi degli $n-1$ differenziali delle $F_{\beta}$, cioè dal sistema di equazioni

$$
\left.\begin{array}{cccc}
F_{1,1} & d z_{1}+F_{1,2} & d z_{2}+\cdots+F_{1, n} & d z_{n}=0 \\
F_{8,1} & d z_{1}+F_{2,2} & d z_{2}+\cdots+F_{2, n} & d z_{n}=0 \\
\ldots & \cdots \cdots \cdots \cdots \cdots \\
F_{n-1,1} & d z_{1}+F_{n-1,2} d z_{2}+\cdots+F_{n-1, n} d z_{n}=0 \\
F_{n, 1} & d z_{1}+F_{n, 2} & d z_{2}+\cdots+F_{n, n} & d z_{n}=d F_{n}
\end{array}\right\}
$$

Questo puo riguardarsi come un sistema di equazioni differenziali ordinarie, per il quale le $n$ grandezze $z_{\alpha}$ dipendono dalla variabile indipendente $F_{n}$. La risoluzione algebrica delle equazioni (34) dả pei quozienti differenziali $\frac{d z}{d \bar{F}}$, poichè il determinante funzionale $\Delta$ non puo mai divenire uguale a zero, le espressioni finite a un sol valore

$$
\frac{d z_{\alpha}}{d F_{n}}=\frac{1}{\Delta} \frac{\partial \Delta}{\partial F_{n, \alpha}}
$$

Bisogna determinare le grandezze $z_{\alpha}$ in modo che, mentre la variabile $F_{n}$ va dal valore $X_{n}(0)$ al valore $X_{n}$, esse variino con continuità, e per il va- 
Lipschitz: Della inversione di un sistema di funzioni. 255

lore $F_{n}=X_{n}(0)$ sodisfacciano alle equazioni $z_{\alpha}=z_{\alpha}(0)$. Allora il sistema di valori $\left[z_{1}(1), z_{2}(1), \ldots z_{n}(1)\right]$ che sodisfà alle equazioni (33) è quello che corrisponde al valore $F_{n}=X_{n}$.

Il problema così espresso è contenuto nel problema generale: date in un

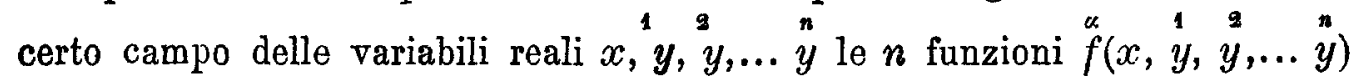
a un sol valore, reali, finite e continue, integrare il sistema di equazioni differenziali

$$
\frac{d \stackrel{\alpha}{y}}{d x}=\stackrel{\alpha}{f}(x, \stackrel{1}{y}, \stackrel{2}{y}, \ldots, \stackrel{n}{y})
$$

in modo che le $n$ funzioni $\stackrel{1}{y}, \frac{2}{y}, \ldots \stackrel{n}{y}$ variino con continuità mentre la variabile indipendente $x$ percorre un certo cammino finito, e per il valore $x=x_{0}$ contenuto in questo cammino prendano valori dati $\stackrel{\alpha}{y=} \stackrel{\alpha}{y}_{0}$. In una ricerca che io ho intrapresa in connessione col presente lavoro, ma che ho pubblicata indipendentemente (*) si deduce, sotto certe condizioni di continuità, per mezzo di un processo graduato, un sistema di funzioni $\stackrel{1}{y}, 2, \ldots, y$. che sodisfä alle poste condizioni colla precisione che si vuole. Sia poi $\stackrel{\alpha}{y}=\stackrel{\alpha}{=}$ una tale soluzione; si spezzi l'intervallo della variabile esteso da $x_{0}$ a $x_{0}+A$ interpolando nell'ordine della loro grandezza i valori $x_{1}, x_{2}, \ldots x_{p-1}$; denoti $\varepsilon_{a}$ una frazione propria, positiva 0 negativa, $\lambda$ una data grandezza positiva, piccola quanto si vuole; il numero a vada da 1 a $p-1$; il sistema di valori $\stackrel{\alpha}{y}=\stackrel{\alpha}{Y}_{a}$ appartenga al valore $x=x_{a}$, e si abbia la serie di equazioni

$$
\stackrel{\alpha}{Y}_{a+1}-\stackrel{\alpha}{Y}_{a}=\left[f^{\alpha}\left(x_{a}, \stackrel{1}{Y}_{a}, \stackrel{2}{Y}_{a}, \ldots \stackrel{\text { m }}{Y}_{a}\right)+\stackrel{\alpha}{\varepsilon_{a}} \lambda\right]\left(x_{a+1}-x_{a}\right)
$$

Allora in quel lavoro è dimostrato che il sistema $\stackrel{\alpha}{Y}$ è identico col sistema a menzionato di sopra. Coerentemente alle osservazioni fatte sopra per le equazioni (24) e $\left(24^{*}\right)$, io formulo il verificarsi della precedente serie di equazioni espressamente come una condizione. Nella integrazione dei sistemi di equazioni differenziali ordinarie, che si considerano nei lavori da me pubblicati, è supposto che questa condizione è sodisfatta.

(*) Disamina della possibitità d'integrare completamente un sistema dato di equazioni differenziali ordinarie: Annali di Matematica, serie $2^{2}$, t. 2, pag. 288. 
Le condizioni di continuità esposte per le funzioni $\stackrel{\alpha}{f}(x, \stackrel{1}{y}, \stackrel{2}{y}, \ldots \stackrel{n}{y})$ sono sempre sodisfatte, perchè esse hanno le loro derivate prime rispetto alle variabili $y, y, \ldots y$ a un sol valore, finite e continue $(*)$; anche queste derivate debbono possedere le proprietà sopra esposte per le equazioni (24*). Nell'applicazione al sistema di equazioni differenziali (35) si fa manifesto che questa supposizione è una conseguenza delle condizioni che sono state poste prima. Infatti le derivate prime rapporto alla variabile delle espressioni $\frac{1}{\Delta} \frac{d \Delta}{d F_{n, \alpha}}$ rapporto alla variabile $z_{\delta}$ sono composte colle derivate prime e seconde delle funzioni $F_{\gamma}$ rapporto alla variabile $z_{\delta}$, e per queste derivate delle funzioni $F_{\gamma}$ è stata fissata nell'art. 2 la proprietà della quale è parola. Se le funzioni $F_{\gamma}$ si concepiscono ottenute colla integrazione di differenziali esatti, le condizioni corrispondenti conducono appunto alle derivate parziali seconde. Nelle vigenti relazioni, si ha dunque una rappresentazione del sistema di valori $\left[z_{1}(1), z_{2}(1), \ldots z_{n}(1)\right]$ che sodisfà alle equazioni (33).

Dopo che si è determinato il sistema di valori $\left[z_{1}(1), z_{2}(1), \ldots z_{n}(1)\right]$, si domanda quel sistema di valori $\left[z_{1}(2), z_{2}(2), \ldots z_{n}(2)\right]$ che sodisfà alle $n$ equazioni

$$
F_{1}=X_{1}(0), F_{2}=X_{2}(0), \ldots F_{n-2}=X_{n-2}(0), F_{n-1}=X_{n-1}, \quad F_{n}=X_{n} .
$$

Qui la totalità dei sistemi di valori, per i quali si ha

$$
F_{1}=X_{1}(0), F_{2}=X_{2}(0), \ldots F_{n-2}=X_{n-2}(0), F_{n}=X_{n}
$$

mentre la funzione $F_{n-1}$ prende con continuita i valori da $X_{n-1}(0)$ a $X_{n-1}$, forma una varietà semplice di primo ordine che è caratterizzata dal sistema di equazioni

$$
\begin{aligned}
& F_{1,1} d z_{1}+F_{1,2} d z_{2}+\cdots+F_{1, n} d z_{n}=0 \\
& \ldots \ldots \ldots \ldots \ldots+\ldots \ldots+\ldots \ldots+F_{n-2, n} d z_{n}=0 \\
& F_{n-2,1} d z_{1}+F_{n-2,1} d z_{2}+\cdots+F_{n-1, n} d z_{n}=d F_{n-1} \\
& F_{n-1,1} d z_{1}+F_{n-1,2} d z_{2}+\cdots+F_{n, n} d z_{n}=0 . \\
& F_{n, 1} d z_{1}+F_{n, 2} d z_{2}+\cdots+\cdots+\cdots
\end{aligned}
$$

(*) Nel lavoro citato, pag. 300 . 
Lipschitz: Della inversione di un sistema di funzioni. 257

Questo dả il sistema di equazioni differenziali ordinarie

$$
\frac{d z_{\alpha}}{d F_{n-1}}=\frac{1}{\Delta} \frac{\partial \Delta}{\partial F_{n-1, \alpha}}
$$

dove le grandezze $z_{\alpha}$ variano con continuità quando la variabile indipendente $F_{n-1}$ va dal valore $X_{n-1}(0)$ al valore $X_{n-1}$, e per il valore $F_{n-1}=X_{n-1}(0)$ devono sodisfare alle equazioni $z_{\alpha}=z_{\alpha}(0)$. Della integrazione del sistema (38) vale tutto ciò che si è detto della integrazione del sistema (35), e il sistema cercato $\left[z_{1}(2), z_{2}(2), \ldots z_{n}(2)\right]$ che corrisponde al valore $F_{n-1}=X_{n-1}$, puó, sotto le relative condizioni, riguardarsi come conosciuto.

E chiaro che si puó ripetere il processo indicato e pervenire a una determinazione del sistema $\left(z_{1}, z_{2}, \ldots z_{n}\right)$ che sodisfá alle equazioni (31), colla successiva integrazione di $n$ sistemi di equazioni differenziali ordinarie che hanno la forma (35). Qui ha pero significato essenziale la circostanza, che se in queste integrazioni si muta l'ordine delle funzioni $F_{\alpha}$, muta anche il processo di tutte le operazioni per le quali si ottiene il risultato definitivo. Quindi non è in alcun modo evidente che le determinazioni che si ottengono con i differenti processi conducano allo stesso risultato. Ma poichè la ricerca fatta nel primo articolo dà il modo di conoscere le condizioni sufficienti perchè il sistema (31) determini in modo unico le grandezze $z_{\alpha}$, la supposizione di quelle condizioni dà diritto ad asserire che, per mezzo del processo esposto, le grandezze $z_{1}, z_{2}, \ldots z_{n}$ vengono rappresentate nella loro unica dipendenza dai valori $X_{1}, X_{2}, \ldots X_{n}$; nel che consisteva appunto lo scopo di questo processo.

\section{5.}

Ora applichiamo le considerazioni esposte ad un sistema di funzioni complesse. Sia il numero fin qui denotato con $n$ uguale a un numero pari $2 \mathrm{~m}$, sia $i$ uguale alla unità imaginaria $V \overline{-1}$, gl'indici $p, q, \ldots$ trascorrano la serie dei numeri da 1 ad $m$, e tanto due funzioni $F_{\alpha}$ quanto due variabili $z_{\beta}$ siano combinate per mezzo delle equazioni

$$
f_{p}=F_{p}+i F_{m+p}, \quad y_{q}=z_{q}+i z_{m+q} \text {. }
$$

Affinchè una espressione $f_{p}$ sia una funzione complessa delle $m$ variabili complesse $y_{q}$, è necessario e sufficiente che tra i differenziali

$$
d f_{p}=d F_{p}+i d F_{n+2}, \quad d y_{q}=d z_{q}+i d z_{m+q}
$$


si abbia la equazione

$$
d f_{p}=\mathbf{\Sigma}_{q} f_{p, q} d y_{q}
$$

dove le $f_{p, q}$ denotano espressioni complesse, finite, dipendenti dalle variabili $z_{1}, z_{2}, \ldots z_{n}$. Se questa relazione si confronta colla relazione che ha luogo in generale

$$
\left.\begin{array}{l}
d f_{p}=d F_{p}+i d F_{m+p}= \\
\quad=\Sigma_{q}\left(\frac{\partial F_{p}}{\partial z_{q}}+i \frac{\partial F_{m+p}}{\partial z_{q}}\right) d z_{q}+\Sigma_{q}\left(\frac{\partial F_{p}}{\partial z_{m+q}}+i \frac{\partial F_{m+p}}{\partial z_{m+q}}\right) d z_{m+q}
\end{array}\right\}
$$

si ha il sistema di relazioni

$$
f_{p, q}=\frac{\partial F_{p}}{\partial z_{q}}+i \frac{\partial F_{n+p}}{\partial z_{q}}=-i\left(\frac{\partial F_{p}}{\partial z_{m+q}}+i \frac{\partial F_{m+p}}{\partial z_{m+q}}\right)
$$

che reciprocamente ha per conseguenza l'equazione (40).

Supporremo che per ciascuna delle espressioni $\rho_{q}$ abbia luogo la relativa equazione (40), ossia che le espressioni $f_{q}$ formino un sistema di funzioni delle variabili complesse $y_{1}, y_{2}, \ldots y_{m}$. Ora la soluzione algebrica delle $m$ equazioni (40) dia il sistema di determinazioni

$$
d y_{q}=\Sigma_{p} y_{q, p} d f_{p}
$$

Se qui è permesso di riguardare le grandezze reali $z_{1}, z_{2}, \ldots . z_{2 m}$ come funzioni dei valori reali $F_{1}, F_{2}, \ldots F_{2 m}$, le espressioni $y_{q, p}$ divengono funzioni dei valori $F_{1}, F_{2}, \ldots F_{2 m}$, e le equazioni (43) esprimono il fatto, che le grandezze $y_{q}$ sono funzioni complesse delle grandezze complesse $f_{p}$. Quindi, tostoche per mezzo delle condizioni sviluppate nei primi articoli, le grandezze reali $z_{1}, z_{2}, \ldots z_{2 m}$ sono funzioni a un sol valore dei valori reali $f_{1}$, $f_{2}, \ldots f_{2 m}$, si è in diritto d'invertire questa relazione per il sistema di funzioni $f_{1}, f_{2}, \ldots f_{2 m}$ delle variabili complesse $y_{1}, y_{2}, \ldots y_{2 m}$, e di considerare le grandezze $y_{1}, y_{2}, \ldots y_{m}$ come funzioni complesse a un sol valore delle variabili complesse $f_{1}, f_{2}, \ldots f_{m}$.

Per formare in questo caso il determinante funzionale $\Delta$ delle funzioni reali $F_{1}, F_{2}, \ldots F_{2 m}$ rapporto alle variabili reali $z_{1}, z_{2}, \ldots z_{2 m}$, denotiamo con $f_{p}^{\prime}, y_{q}^{\prime}, f_{p, q}^{\prime}$ le espressioni rispettivamente coniugate ad $f_{p}, y_{q}, f_{p, q}$. Dalla (39*) si hanno allora le equazioni

$$
d f_{p}^{\prime}=d F_{p}-i d F_{m+p}, \quad d y_{q}^{\prime}=d z_{q}-i d z_{m+q}
$$

e dalla (40) le equazioni

$$
d f_{p}^{\prime}=\mathbf{\Sigma}_{q} d f_{p, q}^{\prime} d y_{q}^{\prime} .
$$


Ora tra $\mathrm{i}$ differenziali introdotti sussistono tre sistemi di $2 m$ equazioni lineari; per mezzo del primo si esprimono $d F_{p}, d F_{m+p}$ per $d f_{p}, d f_{p}^{\prime}$, per mezzo del secondo $d f_{p}, d f_{p}^{\prime}$ per $d y_{q}, d y_{q}^{\prime}$, per mezzo del terzo $d y_{q}, d y_{q}^{\prime}$ per $d z_{q}, d z_{m+q}$. Ciascuno di questi sistemi di equazioni lineari ha un proprio determinante, e per teoremi noti si ha che il determinante funzionale cercato $\Delta$ e uguale al prodotto di questi tre determinanti. Denotiamo con $\left|f_{p, q}\right|$ il determinante dell'equazioni (40), con $\left|f_{p, q}^{\prime}\right|$ quello dell'equazioni (45), sarà facile a vedersi che per $\mathrm{i}$ tre menzionati sistemi di $2 m$ equazioni $\mathrm{i}$ determinanti del primo e del terzo hanno valori reciproci, mentre il determinante del secondo è uguale al prodotto $\left|f_{p, q}\right| \cdot\left|f_{p, q}^{\prime}\right|$. Dunque il prodotto dei tre determinanti è uguale al secondo di essi, e per il determinante funzionale $\Delta$ si ha la espressione

$$
\Delta=\left|f_{p, q}\right| \cdot\left|f^{\prime}{ }_{p, q}\right| \text {. }
$$

Separando nel determinante $\left|f_{p, q}\right|$ le parti reale e imaginaria, essendo $\left|f_{p, q}^{\prime}\right|$ coniugato ad $\left|f_{p, q}\right|$, si ha

$$
\left.\left|f_{p, q}\right|=A+B i, \quad \mid f_{p, q}^{\prime}\right\rfloor=A-B i
$$

e quindi :

$$
\Delta=A^{8}+B^{2} .
$$

Dunque il determinante funzionale $\Delta$ è la somma di due quadrati, e percio essenzialmente positivo, come ha osservato anche il signor Kronecker (*).

Se le grandezze $z_{1} ; z_{2}, \ldots z_{2 m}$, in virtù delle condizioni sopra esposte, debbono essere funzioni a un sol valore delle variabili $F_{1}, F_{2}, \ldots F_{2 m}$, net campo $G$ il determinante funzionale $\Delta$ deve avere da per tutto lo stesso segno. Nel caso presente $\Delta$ non puó divenir mai negativo, e pud annullarsi soltanto quando le parti reali $A$ e $B$ contemporaneamente si annnullano. Dunque nel campo $G$ non possono essere contenuti quei sistemi di valori per i quali avvien questo accidente. La totalità dei relativi sistemi di valori non puo mai formare altro che una varietà $(2 m-2)^{\text {upla }}$. Nel caso $2 m=2$, questi sistemi di valori debbono essere, come è noto, valori singolari.

Bonn, 6 ottobre, 1870.

(*) Nel luogo citato, pag. $17 \%$. 